Running head: Age-related brain asymmetry reduction and interhemispheric integration

(Please note that this manuscript is currently under peer review.)

\title{
White-matter microstructure of the splenium modulates right hemisphere ERP correlates of syntactic processing in older adults
}

Po-Heng Chen ${ }^{1}$, MA, Wan-Ting Lin², MSc, Jen-Shiang Wong ${ }^{3}$, MSc, Wen-Yih Isaac Tseng ${ }^{3,4}, \mathrm{MD}$, PhD, Joshua Oon Soo Goh ${ }^{2,5,6}, \mathrm{PhD}, \&$ Chia-Lin Lee ${ }^{1,2,5,6}$, PhD

1. Graduate Institute of Linguistics,

2. Graduate Institute of Brain and Mind Sciences,

3. Institute of Medical Device and Imaging,

4. Molecular Imaging Center,

5. Department of Psychology,

6. Neurobiology and Cognitive Neuroscience Center,

National Taiwan University, Taipei, Taiwan.

(This manuscript contains 4969 words, 50 references, 1 table, and 2 figures. There are also supplementary materials with 1 table and 3 figures.)

Corresponding author:

Chia-Lin Lee (chialinlee@ntu.edu.tw)

National Taiwan University

Address: No. 1, Sec. 4, Roosevelt Road

Taipei, 10617 Taiwan (R.O.C.) 


\begin{abstract}
Objectives

The present study investigated how increased right-hemisphere (RH) responses in healthy older adults during syntactic processing, a highly left-lateralized function in younger adults, are modulated by callosal integrity.

Methods
\end{abstract}

Syntactic phrase violations in Taiwan Mandarin were presented to either the left or right visual field with event-related potentials (ERPs) measured. Individual participant's microstructural tissue integrity of the corpus callosum was measured with diffusion tensor imaging (DTI).

$\underline{\text { Results }}$

Younger and older adults elicited left-hemisphere (LH) P600 responses to grammatical violations, but only older adults additionally elicited a RH P600 grammaticality effect. Larger RH P600 effects in older adults were associated with larger fractional anisotropy (FA) values of the splenium. This association was not observed for the genu, which evinced significantly lower FA in older relative to younger adults.

\title{
Discussion
}

These results highlight that LH-similar syntactic responses are recruited in older adult RH even for the Mandarin language where syntactic information is routinely accessible via semantic cues. Critically, splenium integrity modulates how older individuals engage inter-hemispheric coordination for syntactic information processing.

\section{Keywords}

aging, functional lateralization, corpus callosum, P600, diffusion tensor imaging 


\section{Introduction}

Functional hemispheric lateralization is one of the hallmarks of human language processing in the brain, amongst which left-hemisphere (LH) syntactic processing is the most robust (Fedorenko et al., 2012; Tyler et al., 2011; Zaccarella \& Friederici, 2017). With aging, however, syntax processing often additionally engages right hemispheric (RH) activity (see Shafto \& Tyler, 2014; Wingfield \& Grossman, 2006 for reviews), echoing hemispheric de-specialization across a broad range of cognitive processes in older adults (see Cabeza, 2002; Goh, 2011 for reviews). Studies have linked older adult functional bi-lateralization with the status of corpus callosal (CC) connections pivotal in inter-hemispheric communication (see Ocklenburg et al., 2016 for a review). At present, however, whether the additional RH involvement in the context of older adult syntax processing reflects inter-hemispheric coordination limited or afforded by $\mathrm{CC}$ white-matter integrity remains inconclusive. To this end, we used diffusion tensor imaging (DTI) and event-related potentials (ERP) in a divided visual field (VF) paradigm to investigate how older adult RHrelated syntax processing is modulated by $\mathrm{CC}$ integrity.

Imaging and lesion studies consistently demonstrate the primary involvement of the left frontotemporal network for syntactic information processing in younger adults (Fedorenko et al., 2012; Griffiths et al., 2012; Zaccarella \& Friederici, 2017), with cooccurring RH involvement reflecting more general semantic operations (Wright et al., 2012). ERP studies of syntactic processing report converging findings based on the divided VF paradigm and the P600 effect, a sustained posterior positivity beginning around $500 \mathrm{~ms}$ after ungrammatical stimuli onsets (Leckey \& Federmeier, 2017; Lee \& Federmeier, 2015; Weng \& Lee, 2020; Yeh et al., 2020). In younger adults, the P600 effect is seen at the group level when grammatical errors are presented to the 
right visual field (RVF), which preferentially engages LH processing, but not when grammatical errors are presented to the left visual field (LVF), which prioritizes the RH. Rather, grammatical errors presented to the LVF elicit N400 effects due to the lexical-semantic unexpectedness of grammatical errors (Kutas \& Federmeier, 2011). Importantly, in both imaging and ERP studies, older adults robustly show more bilateral hemispheric engagement for syntactic processing (Leckey \& Federmeier, 2017; Tyler, Shafto, et al., 2010).

At this juncture, it is important to note that older adult RH syntactic processing is a feature observed in Indo-European language processing (e.g., English or German) and its ubiquity in alternative linguistic contexts remains to be examined. Whereas languages like English and German encode syntactic information primarily with morphological inflections, some languages place greater emphasis on semantic or pragmatic contexts to also convey syntactic relations. For instance, in Mandarin, sparse morpho-syntactic markings and highly flexible word orders result in the prioritizing of semantic (e.g., animacy) over structural cues (e.g., word order) for coding grammatical agent-patient relations (Li et al., 1993). Thus, compromises in processing Mandarin grammatical word class information might be bypassed by meaning-related processes in either hemisphere. For such language speakers, whether increased syntax-related $\mathrm{RH}$ activity is present in older adults is an open question.

Critically, while indices of the integrity of CC white-matter subserving LHRH functional communication generally show declines with age (Bartzokis et al., 2004; Salat et al., 2005; Tseng et al., 2021), mixed findings surround the association between CC integrity declines and hemispheric functional bi-lateralization in older adults. One study showed that greater frontal $\mathrm{CC}$ fractional anisotropy (FA; an integrity index) in older adults was associated with greater frontal functional 
bilaterality, which was in turn associated with cross-hemispheric semantic performance advantage (Davis et al., 2012). However, a longitudinal study in older adults found instead that increasing frontal functional bilaterality was associated with increased task-switching response times and decreases in frontal CC FA (Hakun et al., 2015). These findings suggest that the association between decreases in CC FA and functional bi-lateralization may be process specific.

The association between CC integrity decline and age-related bi-lateralization is further complicated by the differential associations between $\mathrm{CC}$ subregions with lateralization patterns as well as their differential susceptibility to aging. In younger adults, larger general CC mid-sagittal area is associated with more left lateralized language processing (Josse et al., 2008). By contrast, greater mid-sagittal area of the splenium (but not the isthmus, mid-region, and genu of the $\mathrm{CC}$ ) is associated with less language lateralization and better verbal fluency in female adults (Hines et al., 1992). Furthermore, age-related decline in CC structure is not uniform and frontal, latermyelinating, regions (i.e., genu) are more vulnerable than posterior, earlymyelinating, regions (i.e., splenium) (Bartzokis et al., 2004). These results thus highlight the need to investigate the influences of structural integrity decline on hemispheric functional lateralization in subregions of $\mathrm{CC}$ with specific measures that reflect the hypothesized bi-lateralized processing.

In this present study, younger and older Taiwan Mandarin speaking adults contributed DTI data on CC integrity. These same participants also underwent an ERP experiment in which they comprehended phrase structure violations (e.g., nouns appearing in a verb context and vice versa) presented laterally via a divided VF paradigm to selectively bias syntactic affordance to each hemisphere. We expected younger adults to show unilateral RVF/LH P600 and bilateral N400 grammaticality 
effects replicating LH dominance in syntax processing in Taiwan Mandarin. With Mandarin-speaking older adults, detection of bilateral LVF/RH P600 and RVF/LH P600 grammaticality effects would replicate findings based on Indo-European languages. Alternatively, it is possible that Mandarin-speaking older adults might engage accentuated LVF/RH N400 with unilateral RVF/LH P600 grammaticality effects, reflecting added semantic-related contributions when reconciling syntactic relations characteristic of the Mandarin language. Critically, we interrogated how individual differences in the older adult ERP responses to syntactic violations would be associated with DTI indications of white-matter integrity in the genu and splenium. Greater RH grammaticality effects corresponding with greater CC FA would suggest that recruitment of processing in the $\mathrm{RH}$ of older adults reflects inter-hemispheric cross-communication. By contrast, greater $\mathrm{RH}$ effects corresponding with lower CC FA would indicate that RH involvement in such older adults is more disinhibitory in nature.

\section{Methods}

\subsection{Participants}

Forty-eight older and 37 younger adults participated in the grammaticality judgment ERP experiment and were remunerated with cash. Data from 13 older and 2 younger adults were excluded due to excessive artifacts in ERP data, leaving data from 35 older adults ( 9 males; $61-78$ years old, $M=69$ ) and 35 younger adults (17 males; 20-29 years old, $M=23$ ) in final analyses. A subset of 20 older adults (6 males; 61-78 years old, $M=69$ ) and 24 younger adults (12 males; 20-29 years old, $M$ $=23$ ) additionally participated in the DTI session within six months of the ERP session. The sample size was determined based on prior studies exploiting similar 
experimental designs (Leckey \& Federmeier, 2017) or correlational analyses (Davis et al., 2009). See supplementary materials for more details.

\subsection{Materials}

Stimuli were grammatical and ungrammatical two-word Taiwan Mandarin phrases. Each phrase started with a centrally presented syntactic cue to create expectancy for the syntactic category of the following word. Following the syntactic cue, a noun or a verb was presented to either VF, matching or mismatching the syntactic expectancy established by the cue-yielding the Grammatical (e.g., 三張門 票 “three tickets”; 偷偷勾引 “secretly seduce”) and the Ungrammatical condition (e.g., 從未司機 “never driver”; 一份抵達 “one arrive”). Four lists were generated such that across participants, target words were rotated through conditions across VF. Each participant was randomly assigned to one list and viewed 408 phrases in total. See supplementary materials for additional details about the stimuli.

\subsection{ERP experimental procedure}

Participants were seated $100 \mathrm{~cm}$ before a monitor in a testing room. The experiment began with a 15 -trial practice session. At the start of each trial, a plus sign appeared at the center of the screen for $500 \mathrm{~ms}$. After a random stimulus onset asynchrony of between 1000 and $1500 \mathrm{~ms}$, a syntactic cue was presented at the center of the screen for $200 \mathrm{~ms}$. The offset of the cue was followed by a 300-ms interstimulus interval, and then the target word was randomly presented to either the LVF or the RVF for $200 \mathrm{~ms}$. Visual angle from the inner edge of the target to the screen center was kept at 2 degrees (from this point, words subtended additional 2 degrees of horizontal visual angle). See supplementary materials for validation of our visual field manipulation. Participants were instructed to judge the grammaticality of the phrase by pressing a 'yes' or 'no' button upon a probe question “Acceptable?” displayed 
centrally $1000 \mathrm{~ms}$ after the offset of the target word. The probe remained on the screen until participants responded or after a lapse of $2500 \mathrm{~ms}$. The next trial then began after a delay of $1000 \mathrm{~ms}$. A small red dot was presented a few pixels below the screen center throughout to help participants fixate at the center and avoid orienting to the laterally presented targets. Response hand for 'yes' was counterbalanced across participants. The entire experiment was equally divided into 6 blocks and lasted for about 40 minutes.

2.4. EEG recording, data analysis, and component measurement

The electroencephalogram (EEG) was recorded using 32 sintered Ag/AgCL electrodes from the 10-20 system (QuickCap, Neuromedical Supplies, Sterling, TX, USA). All scalp electrodes were online referenced to the vertex reference and rereferenced offline to the average of right and left mastoids. Vertical eye movements were recorded via a pair of electrodes placed on the supraorbital and infraorbital ridges of the left eye; horizontal eye movements were recorded via electrodes at the outer canthus of each eye in a bipolar montage. Electrode impedance was kept below $5 \mathrm{k} \Omega$. The continuous EEG was amplified by the SYNAMPS2 amplifiers (Compumedics Neuroscan, Inc., El Paso, Texas, USA) through a bandpass filter of 0.05-100 $\mathrm{Hz}$ and digitized at a sampling rate of $1000 \mathrm{~Hz}$.

EEG data were segmented from $200 \mathrm{~ms}$ before to $1100 \mathrm{~ms}$ after stimulus onset. Trials contaminated by artifacts from amplifier blocking, signal drift, muscle activity, eye blinks, or excessive eye movements were rejected offline before averaging. Trial loss averaged $25.6 \%$ and $27.87 \%$ for younger and older participants, respectively, a substantial amount of which were due to horizontal eye movements. Artifact-free ERPs were averaged by stimuli type after subtraction of the $200 \mathrm{~ms}$ pre- 
stimulus baseline. Only ERP data for correct trials were subject to statistical analysis. Prior to measurement, ERPs were digitally filtered with a low-pass of $30 \mathrm{~Hz}$.

ERPs were analyzed with omnibus repeated-measures ANOVAs for the P600 effects and, for comparisons with prior findings, the N400 effects. The N400 responses were quantified as the mean amplitudes of data measured between a typical N400 time window-300 to $600 \mathrm{~ms}$ (Kutas \& Federmeier, 2011). Note that the temporal and spatial overlap from the N400 responses inevitably affect the mean amplitudes of the subsequent positive P600 responses. Thus, we quantified the P600 responses by measuring the amount of positive-going deviation from the N400 (see supplementary materials for more details about this approach). Significance levels of correlational analyses were corrected for multiple comparisons using the false discovery rate method with Benjamini-Hochberg adjusted P values reported.

\subsection{MRI Scanning Parameters}

Brain images were acquired on a 3T Siemens Skyra scanner (Siemens, Erlangen, Germany) with a 32-channel head coil. For each participant, DTI images were acquired with repetition time $(\mathrm{TR})=6800 \mathrm{~ms}$, echo time $(\mathrm{TE})=109 \mathrm{~ms}$, flip angle $=90^{\circ}, 49$ axial slices, $2.5 \times 2.5 \times 2.5 \mathrm{~mm}$ resolution, slice gap $=0 \mathrm{~mm}, 78 \times 78$ matrix, two repetitions of 30 diffusion-gradient directions with the b-value of 1000 $\mathrm{mm}^{2} / \mathrm{ms}$, and two b0 images (null direction baseline). We also acquired highresolution T1-weighted images using a 3-dimensional magnetization prepared rapid acquisition gradient echo sequence with $\mathrm{TR}=2000 \mathrm{~ms}, \mathrm{TE}=2.98 \mathrm{~ms}$, flip angle $=9$ degrees, $256 \times 256 \times 192$ matrix, and $1 \times 1 \times 1 \mathrm{~mm}$ resolution for spatial normalization to standard (Montreal Neurological Institute, MNI) template space.

\subsection{DTI analysis}


DTI image processing was conducted using DSI Studio (http://dsi-

studio.labsolver.org/) and Matlab (MathWorks, Natick, MA) running SPM 12

(Statistical Parametric Mapping, Wellcome Trust Centre for Neuroimaging, UK). For each participant, DTI images were corrected for motion artifacts via eddy current correction and coregistered to the b0 image. Next, the 30 direction DTI images were used to derive whole-brain mapping of fractional anisotropy (FA) that estimates directionality of water diffusion per voxel as the proxy for white-matter structural integrity in this present study (Salat et al., 2005). We obtained genu and splenium white-matter tract ROIs from the NTU-DSI-122 template; as defined in previous studies, these ROIs were the bundles of commissural fibers connecting the left and right orbitofrontal gyri and occipital lobes, respectively (Chen et al., 2015; Hsu et al., 2015). NTU-DSI-122 is a high-resolution template of diffusion spectrum images (DSI) in the MNI space acquired in 122 right-handed Taiwanese young adults. To apply these CC ROIs, we generated a study-specific template by spatially normalizing participant T1 images to the MNI space using the DARTEL procedure (Diffeomorphic Anatomical Registration Through Exponentiated Lie algebra) (Ashburner, 2007), which accounted for anatomical differences between younger and older adult brain structures. The resulting deformation parameters were then applied to transform template genu and splenium tract ROI coordinates from the NTU-DSI122 space to each subject's DTI image space. Tract-Based Automatic Analysis (Chen et al., 2015; Hsu et al., 2015) was then used to sample FA estimates over 100 steps along the length of each white-matter tract ROI applied on each participant's FA image in native subject space. For the purposes of the study, the 100 steps of tract FA estimates were averaged to yield mean FA values summarizing the white-matter 
integrity of the $\mathrm{CC}$ genu and splenium tracts, which were then submitted to further analysis against ERP measures.

\section{Results}

\subsection{ERP grammaticality judgment experiment}

\subsubsection{Behavioral results}

Grammaticality judgment behaviors in younger and older adult groups are shown in Table 1. An omnibus ANOVA with a between-subjects factor of Age (young vs. older) and a within-subjects factor of Visual Field (RVF vs. LVF) on mean accuracies jointly across both phrase types revealed a significant main effect of Age, $F(1,68)=$ $24.79, p<0.001$, but no effect of Visual Field or Age-by-Visual Field interaction ( $p$ s $>0.24)$. To further clarify whether these accuracy differences were driven more by reduced judgment of grammatical phrases as grammatical (hits) or increased judgment of ungrammatical phrases as grammatical (false alarms) in older than younger adults, we conducted a two-sample t-test on the response contrast [false alarms Older $_{-}$false alarms Younger] $_{\text {- [hitsYounger }}$ - hitsolder], collapsing across VFs. This revealed that older adults were significantly less discriminative than younger adults of the grammaticality of ungrammatical compared to grammatical phrases, $\mathrm{t}(66.48)=$ $2.08, p<0.05$.

\section{Table $1 \longrightarrow$}

\subsubsection{ERP results}

Figure 1 illustrates the grand average ERPs elicited by grammatical and ungrammatical critical words with $\mathrm{RVF} / \mathrm{LH}$ and $\mathrm{LVF} / \mathrm{RH}$ presentation for younger and older adults. Descriptively, younger adults elicited more negative N400 responses for ungrammatical relative to grammatical words with both VF presentations, but a 
P600 response with the RVF/LH presentation only. By contrast, older adults elicited biphasic N400-P600 grammaticality effects regardless of visual field of presentation.

\section{- Figure $1 \longrightarrow$}

\subsubsection{N400 effect}

An omnibus ANOVA with Age (young vs. older) as a between-subjects factor and Grammaticality (grammatical vs. ungrammatical) and Visual Field (RVF vs. LVF) as within-subject factors was conducted on the mean amplitudes measured between 300 and $600 \mathrm{~ms}$ over all 30 scalp electrodes. The results showed significant main effects of Age, $F(1,68)=10.31, p<0.01$, Grammaticality, $F(1,68)=23.58, p<$ 0.001 , and Visual Field, $F(1,68)=5.26, p<0.05$. The effect of Grammaticality was modulated by Age, $F(1,68)=6.24, p<0.05$, but not by Visual Field $(\mathrm{p}=0.39)$. Follow-up comparisons showed that the effect of Grammaticality was reliable for younger adults, $F(1,34)=23.59, p<0.001$. For older adults, the N400 effect was only marginal, $F(1,34)=3.26, p=0.08$, but achieved statistical significance when the test was limited to the central-posterior channels $(\mathrm{C} 3, \mathrm{Cz}, \mathrm{C} 4, \mathrm{CP} 3, \mathrm{CPz}, \mathrm{CP} 4, \mathrm{P} 3, \mathrm{Pz}, \mathrm{P} 4$, $\mathrm{O} 1, \mathrm{Oz}, \mathrm{O} 2)$ where N400 effects are typically most prominent, $F(1,34)=4.20, p<$ 0.05 .

\subsubsection{P600 effect}

The same omnibus ANOVA applied on P600 responses revealed reliable effects of Grammaticality, $F(1,68)=29.22, p<0.001$, and Visual Field, $F(1,68)=15.27, p<$ 0.001. Different from the N400 effect, the P600 effect was jointly modulated by both Visual Field and Age, $F(1,68)=5.46, p<0.05$. Planned comparisons within each age group showed that the P600 effects were significant only with the RVF presentation for younger adults, $F(1,34)=25.06, p<0.001$, but were significant with both VF presentations for older adults $(p s<0.01)$. To explore the influence of the RH P600 
effect on behavior, older adults' RH P600 effects were regressed against their syntactic performance. However, no significant correlations were seen either for overall accuracy or separate accuracies for grammatical and grammatical conditions for either visual field $(p s>0.2)$.

\subsection{Age differences in CC integrity in DTI data}

We first examined age-related differences in the genu and splenium tracts by conducting an ANOVA with Age (young vs. older) as a between-subjects factor and CC subregions (genu vs. splenium) as a within-subject factor. The results showed reliable main effects of Age, $F(1,42)=5.08, p<0.05$, and CC subregions, $F(1,42)=$ 6.81, $p<0.05$. Furthermore, the effect of Age interacted with $\mathrm{CC}$ subregions, $F(1,42)$ $=30.68, p<0.001$. Follow-up comparisons revealed a reliable Age effect for the genu $(p<0.001)$, with lower mean FA values for older adults $(0.50, \mathrm{SD}=0.02)$ relative to younger adults $(0.54, \mathrm{SD}=0.03)$. By contrast, the FA values in the posterior region of the $\mathrm{CC}$, splenium, were not statistically different between younger $(0.51, \mathrm{SD}=0.03)$ and older adults $(0.52, \mathrm{SD}=0.03)(p>0.28)$.

\subsection{Microstructural integrity of the splenium tract associated with older adult} LVF/RH P600 effect

Critically, we examined the relationship between older adult LVF/RH P600 grammaticality effects, which distinguished older from younger syntax violation ERP responses, and the microstructural integrity of the genu and splenium tracts. In our sample of older adults, larger RH P600 effects significantly correlated with greater FA of the splenium tract $(r=0.54, p<0.05)$ (Figure 2$)$. By contrast, the correlation between the RH P600 effect and FA of the genu tract was not significant ( $r=0.03, p$ $=0.89)$. Out of interest, we also correlated younger adults' RH P600 responses with FA values of these two CC tracts, but found no significant associations. In addition, 
neither younger nor older adults showed significant associations between LVF/RH N400 effects and FA values of both CC tracts (Supplementary Figures $2 \& 3$ ).

- Figure $2-$

\section{Discussion}

In this present study, we investigated the nature of increased RH-biased ERP responses in healthy older adults during syntactic processing and how callosal integrity modulated such RH involvement. When judging the grammaticality of twoword phrases with the target word presented to the RVF, LH P600 responses to grammatical violations were seen in both younger and older adults, as expected. By contrast, target words presented to the LVF engaged a RH P600 grammaticality effect in the older adults only. These results indicate that even in Taiwan Mandarin where syntactic relations can be routinely understood with supplementary semantic or pragmatic information, LH-similar syntactic processes (i.e., P600-related) instead of lexical-semantic processes (i.e., N400-related) were recruited in the RH of healthy older adults. Comparisons for $\mathrm{CC}$ structural integrity between the two age groups replicated past findings that genu integrity was compromised in the majority of the older participants with the splenium relatively well-preserved with age in our normative sample. Critically, however, we found that individual differences in splenium rather than genu microstructural integrity distinguished expression of the RH P600 grammaticality effect in older individuals. Such ERP-DTI associations of the splenium suggest an active involvement of resources from the non-dominant hemisphere during syntactic processing that jointly might be more sensitive to older individual differences in cognitive ability as compared to genu integrity.

\subsection{Increased RH P600 responses in older speakers of Taiwan Mandarin during syntactic processing}


Our findings are in line with age-related bi-lateralization seen in syntactic processing in Indo-European languages in past studies (Antonenko et al., 2013; Leckey \& Federmeier, 2017; Tyler, Shafto, et al., 2010) as well as more general cognitive functions (e.g., episodic memory, semantic memory, working memory, perception, and inhibitory control; Cabeza, 2002; Goh, 2011; Reuter-Lorenz \& Park, 2014). These past findings have been equivocal with regards to the nature of older adult increased RH activity during syntactic comprehension. For example, higher inter-hemispheric resting-state functional connectivity between frontal areas is found to correlate with reduced FA and worse performance when older adults comprehend syntactically complex sentences (between the left BA 44 and the right BA 44/45, frontal pole, as well as angular gyrus; Antonenko et al., 2013) and learn dependencies in an artificial grammar (between left and right BA 44/45; Antonenko et al., 2012). These findings were interpreted as weakened cross-hemisphere inhibition on the RH homologue of the syntactic network that is not specialized or involved in the particular task.

Here, we show that the RH P600 response recruited in older adults during syntactic comprehension is qualitatively similar to P600-related syntactic processing in the LH. Critically, while the P600 is not a syntax-specific component, it is a reliable index for capturing online processing differences between ill-formed vs. grammatical and simpler vs. complex syntactic structures (see Leckey \& Federmeier, 2020 for a review). Further, as mentioned, Mandarin is a language wherein morphosyntactic marking is sparse and syntactic relations are routinely understood with semantic and/or pragmatic cues (Li et al., 1993). In this light, our older adult Taiwan Mandarin speakers still manifested RH grammaticality effects in the P600 
component reflecting $\mathrm{LH}$-similar syntactic processing rather than semantic processing indexed by N400-related effects.

\subsection{Higher splenium integrity is associated with larger RH P600 in older adults}

Our finding of a positive correlation between splenium integrity and RH P600 syntactic processing in older adults is consistent with its role in supporting interhemispheric coordination during language-related processing, particularly in the posterior cerebrum (Blaauw \& Meiners, 2020). Indeed, the splenium as the most posterior part of the $\mathrm{CC}$ consists of reciprocal and homotopic connections across posterior brain areas, among which temporal and parietal regions have been implicated in syntactic processing (Rodd et al., 2010). Indices of greater splenium integrity have been associated with faster interhemispheric transfer time (IHTT) (Westerhausen et al., 2006), less hemispheric timing difference during dichotic listening (Friedrich et al., 2017), and greater word production in infants reading achievement (Hynd et al., 1995). Conversely, lesions in posterior CC compromise the ability to coordinate LH-dominant syntactic information and task-relevant information from the RH (in this case prosodic information) (Sammler et al., 2010). Interestingly, better splenium connectivity has been linked with more positive general cognitive aging. Higher splenium integrity is found to predict better verbal as well as non-verbal abilities in younger and older adults (Hines et al., 1992; Penke et al., 2010), older adult verbal memory (Cox et al., 2015), and cognitive gains from memory training (Park et al., 2018).

Current perspectives consider that interhemispheric coordination is in principle slow and costly such that task-relevant time-sensitive processes rely more on faster intra-hemispheric communication instead (see Aboitiz \& Montiel, 2003 for a review). Nevertheless, the more active hemisphere can involve the less active hemisphere 
when task demands require (Weissman \& Banich, 2000). Taking the above together, we suggest that RH P600 in older adults in our study is consistent with compensatory engagement under affordance by splenium integrity.

\subsection{RH compensatory attempt associated with splenium connection?}

Findings in younger adults support the compensatory role of the RH P600 to resolve uncertainty in syntactic information under greater task demands or when grammatical cues are impoverished, such as might be the case in older adult brains with reduced processing resources. For instance, RH P600 grammaticality effect is absent at the group level in young native speakers wherein syntactic processing is proficient and effective (Lee \& Federmeier, 2015; Yeh et al., 2020). However, RH P600 grammaticality effects were observed in a subset of intermediate-to-advanced second language learners with relatively low proficiency (Chen et al., 2018).

Moreover, whereas unilateral LH P600 grammaticality effects are observed in response to violations of more easily learned novel structural rules about pseudoword strings (with high variability of intervening items; Gómez, 2002), bi-hemispheric P600 responses are elicited for violations of rules learned under more challenging conditions (see Lee, 2018 for a review). Interestingly, initial recruitment of the RH is predictive of successful learning of grammar in healthy young adults (Qi \& Legault, 2020).

We note that older adults in this present study showed lower accuracy in grammaticality judgment than younger adults, and older adult accuracies did not significantly correlate with increased RH P600 expression. It is possible that RH processing provides only partial compensation in aging brain operations and does not necessarily adequately replace syntactic function in the LH (Shafto \& Tyler, 2014). For instance, additional right frontotemporal BOLD activity in older adults 
comprehending spoken grammatical sentences did not correlate with older adults' behavioral syntactic performance (Tyler, Shafto, et al., 2010; Tyler, Wright, et al., 2010). Furthermore, as age-related differences in frontal activity to syntactically rare usages were only observed when an active task was required (Davis et al., 2014), these additional RH activities might reflect general task demand rather than the same specific linguistic computations as in LH activations (Shafto \& Tyler, 2014).

In this light, we acknowledge that while the older adult RH P600 grammaticality effect in this present study was qualitatively similar to the LH P600 effect, it is unclear to what extent these $\mathrm{RH}$ responses take up the syntactic function of the LH. At the very least, our findings do distinguish the older adult RH P600 processing of grammatical violations from its more semantic counterpart, the N400. Moreover, considering our stimuli were simple two-word phrases that did not impose high working memory load, our findings likely reflect basic syntactic processing rather than general task demand in older adults. Thus, we suggest that older adults with stronger splenial connectivity were more flexible in incorporating resources from the RH during syntactic processing, albeit the benefit to behavioral judgments were obscured. For example, in addition to syntactic processing, the explicit grammaticality judgment task might have weighed on meta-linguistic and decision-making processes as well. More implicit and fine-tuned measures of syntax processing behavior along with the antecedents and corollaries of the RH P600 grammaticality effects are necessary to clarify these contingencies in future studies.

\subsection{No evidence for a reliable association between genu integrity and RH P600}

The absent association between older adult genu integrity and RH P600 grammaticality effect highlights the more critical role of the splenium in determining cognitive status in aging. Nevertheless, this null finding does not preclude the 
involvement of genu in modulating syntactic performance. Prior studies have found a positive modulation of the FA integrity in tracts connecting left and right Brodmann areas (BA) 44/45 on syntactic performance and artificial grammar learning performance in older adults (Antonenko et al., 2012, 2013). Furthermore, callosal size of both anterior (the rostrum and genu) and posterior (isthmus and splenium) regions have also been associated with ear asymmetry during dichotic listening (Gootjes et al., 2006), suggesting a role of genu in inter-hemispheric processing of linguistic inputs. Note, however, in this present study, genu integrity was significantly lower in older adults relative to younger adults, consistent with a host of neuroimaging findings demonstrating that anterior and frontal areas of the $\mathrm{CC}$ are more vulnerable to normal aging relative to posterior areas (Bartzokis et al., 2004; Salat et al., 2005; Tseng et al., 2021). It is possible that more extensive age-related neurobiological declines in older adult genu resulted in minimal variation in FA associated with individual syntactic performance and RH P600 responses. Overall, the role of genu in mediating syntactic processing resources across hemispheres in older adults requires further studies possibly targeting middle-aged adults or expanding the selection of older adults with more intact genu.

\section{Conclusion}

Our study speaks to how the brain evinces differential functional reorganization in older individuals. We replicate past findings that syntactic processing, which is highly lateralized in young adult $\mathrm{LH}$, additionally engages the $\mathrm{RH}$ in older adults as a group, and extend this effect to Mandarin speakers. Critically, we present novel evidence that expression of such RH syntactic engagement is greater in older adult individuals with greater splenium integrity. Older adults with splenium atrophy might not be able to recruit $\mathrm{RH}$ resources even if the task requires. Studies delineating such 
contingent contribution of inter-hemispheric functional communication bounded by individual brain structural variations are crucial in understanding the dynamical nature of observed aging brain function. 


\section{Funding}

This work was supported by Taiwan Ministry of Science and Technology research grants (\# MOST 106-2420-H-002-006-MY2 and \# MOST 108-2410-H-002-056-MY2 to Chia-Lin Lee).

\section{Acknowledgements}

The authors wish to thank Min-Hsin Chen for stimuli construction and preparation of this experiment as well as Yi-Chun Chen, Ling-Chen Chou, Chia-Ho Lai, Tzu-Hung Lu, Zih-Yu Shen, Ling Tang, Yi-Ting Tsai, and Chih Yeh for assistance with collection and preprocessing of the data. Our data, analytic codes, and research materials will be available on a trusted repository once the manuscript is accepted. This research was not pre-registered. 


\section{References}

Aboitiz, F., \& Montiel, J. (2003). One hundred million years of interhemispheric communication: the history of the corpus callosum. Brazilian journal of medical and biological research, 36(4), 409-420. https://doi.org/10.1590/s0100-879x2003000400002

Antonenko, D., Brauer, J., Meinzer, M., Fengler, A., Kerti, L., Friederici, A. D., \& Flöel, A. (2013). Functional and structural syntax networks in aging. Neuroimage, 83, 513-523. https://doi.org/10.1016/j.neuroimage.2013.07.018

Antonenko, D., Meinzer, M., Lindenberg, R., Witte, A. V., \& Flöel, A. (2012). Grammar learning in older adults is linked to white matter microstructure and functional connectivity. Neuroimage, 62(3), 1667-1674. https://doi.org/10.1016/j.neuroimage.2012.05.074

Ashburner, J. (2007). A fast diffeomorphic image registration algorithm. Neuroimage, 38(1), 95-113. https://doi.org/10.1016/j.neuroimage.2007.07.007

Bartzokis, G., Sultzer, D., Lu, P. H., Nuechterlein, K. H., Mintz, J., \& Cummings, J. L. (2004). Heterogeneous age-related breakdown of white matter structural integrity: implications for cortical "disconnection" in aging and Alzheimer's disease. Neurobiology of aging, 25(7), 843-851. https://doi.org/10.1016/j.neurobiolaging.2003.09.005 
Blaauw, J., \& Meiners, L. C. (2020). The splenium of the corpus callosum: embryology, anatomy, function and imaging with pathophysiological hypothesis. Neuroradiology, 62(5), 563-585. https://doi.org/10.1007/s00234$\underline{019-02357-z}$

Cabeza, R. (2002). Hemispheric asymmetry reduction in older adults: the HAROLD model. Psychology and Aging, 17(1), 85. https://doi.org/10.1037//0882$\underline{7974.17 .1 .85}$

Chen, P.-H., Yeh, C., Lu, C., Hsieh, S.-K., Chou, T.-L., Su, L. I.-W., \& Lee, C.-L. (2018). Multiple scaffolding mechanisms for L2 syntactic processing: An Event-Related Potential study. Journal of Chinese Language Teaching, 15(2), 63-93. http://www.airitilibrary.com/Publication/alDetailedMesh?DocID=18118429$\underline{201806-201807040021-201807040021-63-93}$

Chen, Y.-J., Lo, Y.-C., Hsu, Y.-C., Fan, C.-C., Hwang, T.-J., Liu, C.-M., Chien, Y.L., Hsieh, M. H., Liu, C.-C., Hwu, H.-G., \& Tseng, W.-Y. I. (2015). Automatic whole brain tract-based analysis using predefined tracts in a diffusion spectrum imaging template and an accurate registration strategy. Human brain mapping, 36(9), 3441-3458. https://doi.org/10.1002/hbm.22854

Cox, S. R., Bastin, M. E., Ferguson, K. J., Allerhand, M., Royle, N. A., Maniega, S. M., Starr, J. M., MacLullich, A. M. J., Wardlaw, J. M., Deary, I. J., \& MacPherson, S. E. (2015). Compensation or inhibitory failure? Testing 
hypotheses of age-related right frontal lobe involvement in verbal memory ability using structural and diffusion MRI. Cortex, 63, 4-15. https://doi.org/10.1016/j.cortex.2014.08.001

Davis, S. W., Dennis, N. A., Buchler, N. G., White, L. E., Madden, D. J., \& Cabeza, R. (2009). Assessing the effects of age on long white matter tracts using diffusion tensor tractography. Neuroimage, 46(2), 530-541. https://doi.org/10.1016/j.neuroimage.2009.01.068

Davis, S. W., Kragel, J. E., Madden, D. J., \& Cabeza, R. (2012). The Architecture of Cross-Hemispheric Communication in the Aging Brain: Linking Behavior to Functional and Structural Connectivity. Cerebral Cortex, 22(1), 232-242. https://doi.org/10.1093/cercor/bhr123

Davis, S. W., Zhuang, J., Wright, P., \& Tyler, L. K. (2014). Age-related sensitivity to task-related modulation of language-processing networks. Neuropsychologia, 63, 107-115. https://doi.org/10.1016/j.neuropsychologia.2014.08.017

Fedorenko, E., Nieto-Castañon, A., \& Kanwisher, N. (2012). Lexical and syntactic representations in the brain: An fMRI investigation with multi-voxel pattern analyses. Neuropsychologia, 50(4), 499-513. https://doi.org/10.1016/j.neuropsychologia.2011.09.014

Friedrich, P., Ocklenburg, S., Heins, N., Schlüter, C., Fraenz, C., Beste, C., Güntürkün, O., \& Genç, E. (2017). Callosal microstructure affects the timing 
of electrophysiological left-right differences. Neuroimage, 163, 310-318. https://doi.org/10.1016/j.neuroimage.2017.09.048

Goh, J. O. S. (2011). Functional Dedifferentiation and Altered Connectivity in Older Adults: Neural Accounts of Cognitive Aging. Aging and disease, 2(1), 30-48. http://www.aginganddisease.org/CN/abstract/article_147266.shtml

Gómez, R. L. (2002). Variability and Detection of Invariant Structure. Psychological Science, 13(5), 431-436. https://doi.org/10.1111/1467-9280.00476

Gootjes, L., Bouma, A., Van Strien, J., Van Schijndel, R., Barkhof, F., \& Scheltens, P. (2006). Corpus callosum size correlates with asymmetric performance on a dichotic listening task in healthy aging but not in Alzheimer's disease. Neuropsychologia, 44(2), 208-217. https://doi.org/10.1016/j.neuropsychologia.2005.05.002

Griffiths, J. D., Marslen-Wilson, W. D., Stamatakis, E. A., \& Tyler, L. K. (2012). Functional Organization of the Neural Language System: Dorsal and Ventral Pathways Are Critical for Syntax. Cerebral Cortex, 23(1), 139-147. https://doi.org/10.1093/cercor/bhr386

Hakun, J. G., Zhu, Z., Brown, C. A., Johnson, N. F., \& Gold, B. T. (2015). Longitudinal alterations to brain function, structure, and cognitive performance in healthy older adults: A fMRI-DTI study. Neuropsychologia, 71, 225-235. https://doi.org/10.1016/j.neuropsychologia.2015.04.008 
Hines, M., Chiu, L., McAdams, L. A., Bentler, P. M., \& Lipcamon, J. (1992).

Cognition and the corpus callosum: verbal fluency, visuospatial ability, and language lateralization related to midsagittal surface areas of callosal subregions. Behavioral neuroscience, 106(1), 3. https://doi.org/10.1037//07357044.106.1.3

Hsu, Y. C., Lo, Y. C., Chen, Y. J., Wedeen, V. J., \& Tseng, W.-Y. I. (2015). NTUDSI-122: A diffusion spectrum imaging template with high anatomical matching to the ICBM-152 space. Human brain mapping, 36(9), 3528-3541. https://doi.org/10.1002/hbm.22860

Hynd, G. W., Hall, J., Novey, E. S., Eliopulos, D., Black, K., Gonzalez, J. J., Edmonds, J. E., Riccio, C., \& Cohen, M. (1995). Dyslexia and Corpus Callosum Morphology. Archives of Neurology, 52(1), 32-38. https://doi.org/10.1001/archneur.1995.00540250036010

Josse, G., Seghier, M. L., Kherif, F., \& Price, C. J. (2008). Explaining Function with Anatomy: Language Lateralization and Corpus Callosum Size. The Journal of Neuroscience, 28(52), 14132. https://doi.org/10.1523/JNEUROSCI.4383$\underline{08.2008}$

Kutas, M., \& Federmeier, K. D. (2011). Thirty Years and Counting: Finding Meaning in the N400 Component of the Event-Related Brain Potential (ERP). Annual 
review of psychology, 62(1), 621-647.

https://doi.org/10.1146/annurev.psych.093008.131123

Leckey, M., \& Federmeier, K. D. (2017). Age-related shifts in hemispheric dominance for syntactic processing. Psychophysiology, 54(12), 1929-1939. https://doi.org/10.1111/psyp.12970

Leckey, M., \& Federmeier, K. D. (2020). The P3b and P600(s): Positive contributions to language comprehension. Psychophysiology, 57(7), e13351. https://doi.org/10.1111/psyp.13351

Lee, C. L. (2018). Familial Sinistrality and Language Processing. In K. D. Federmeier \& D. G. Watson (Eds.), Psychology of Learning and Motivation (Vol. 68, pp. 143-177). Academic Press. https://doi.org/10.1016/bs.plm.2018.08.005

Lee, C. L., \& Federmeier, K. D. (2015). It's All in the Family: Brain Asymmetry and Syntactic Processing of Word Class. Psychological Science, 26(7), 997-1005. https://doi.org/10.1177/0956797615575743

Li, P., Bates, E., \& Macwhinney, B. (1993). Processing A Language without Inflections: A Reaction Time Study of Sentence Interpretation in Chinese. Journal of Memory and Language, 32(2), 169-192. https://doi.org/10.1006/jmla.1993.1010 
Ocklenburg, S., Friedrich, P., Güntürkün, O., \& Genç, E. (2016). Intrahemispheric white matter asymmetries: the missing link between brain structure and functional lateralization? Reviews in the Neurosciences, 27(5), 465-480. https://doi.org/10.1515/revneuro-2015-0052

Park, S., Ryu, S.-H., Yoo, Y., Yang, J.-J., Kwon, H., Youn, J.-H., Lee, J.-M., Cho, S.J., \& Lee, J.-Y. (2018). Neural predictors of cognitive improvement by multistrategic memory training based on metamemory in older adults with subjective memory complaints. Scientific Reports, 8(1), 1095. https://doi.org/10.1038/s41598-018-19390-2

Penke, L., Maniega, S. M., Houlihan, L. M., Murray, C., Gow, A. J., Clayden, J. D., Bastin, M. E., Wardlaw, J. M., \& Deary, I. J. (2010). White matter integrity in the splenium of the corpus callosum is related to successful cognitive aging and partly mediates the protective effect of an ancestral polymorphism in ADRB2. Behavior genetics, 40(2), 146-156. https://doi.org/10.1007/s10519$\underline{009-9318-4}$

Qi, Z., \& Legault, J. (2020). Neural hemispheric organization in successful adult language learning: Is left always right? In K. D. Federmeier \& H.-W. Huang (Eds.), Psychology of Learning and Motivation (Vol. 72, pp. 119-163). Academic Press. https://doi.org/10.1016/bs.plm.2020.02.004 
Reuter-Lorenz, P. A., \& Park, D. C. (2014). How Does it STAC Up? Revisiting the Scaffolding Theory of Aging and Cognition. Neuropsychology review, 24(3), 355-370. https://doi.org/10.1007/s11065-014-9270-9

Rodd, J. M., Longe, O. A., Randall, B., \& Tyler, L. K. (2010). The functional organisation of the fronto-temporal language system: evidence from syntactic and semantic ambiguity. Neuropsychologia, 48(5), 1324-1335. https://doi.org/10.1016/j.neuropsychologia.2009.12.035

Salat, D., Tuch, D., Greve, D., Van Der Kouwe, A., Hevelone, N., Zaleta, A., Rosen, B., Fischl, B., Corkin, S., \& Rosas, H. D. (2005). Age-related alterations in white matter microstructure measured by diffusion tensor imaging. Neurobiology of aging, 26(8), 1215-1227. https://doi.org/10.1016/j.neurobiolaging.2004.09.017

Sammler, D., Kotz, S. A., Eckstein, K., Ott, D. V., \& Friederici, A. D. (2010). Prosody meets syntax: the role of the corpus callosum. Brain, 133(9), 26432655. https://doi.org/10.1093/brain/awq231

Shafto, M. A., \& Tyler, L. K. (2014). Language in the aging brain: the network dynamics of cognitive decline and preservation. Science, 346(6209), 583-587. https://doi.org/10.1126/science.1254404

Tseng, W.-Y. I., Hsu, Y.-C., Chen, C.-L., Kang, Y.-J., Kao, T.-W., Chen, P.-Y., \& Waiter, G. D. (2021). Microstructural differences in white matter tracts across 
middle to late adulthood: a diffusion MRI study on 7167 UK Biobank participants. Neurobiology of aging, 98, 160-172.

https://doi.org/10.1016/j.neurobiolaging.2020.10.006

Tyler, L. K., Marslen-Wilson, W. D., Randall, B., Wright, P., Devereux, B. J., Zhuang, J., Papoutsi, M., \& Stamatakis, E. A. (2011). Left inferior frontal cortex and syntax: function, structure and behaviour in patients with left hemisphere damage. Brain, 134(2), 415-431.

https://doi.org/10.1093/brain/awq369

Tyler, L. K., Shafto, M. A., Randall, B., Wright, P., Marslen-Wilson, W. D., \& Stamatakis, E. A. (2010). Preserving syntactic processing across the adult life span: the modulation of the frontotemporal language system in the context of age-related atrophy. Cerebral Cortex, 20(2), 352-364. https://doi.org/10.1093/cercor/bhp105

Tyler, L. K., Wright, P., Randall, B., Marslen-Wilson, W. D., \& Stamatakis, E. A. (2010). Reorganization of syntactic processing following left-hemisphere brain damage: does right-hemisphere activity preserve function? Brain, 133(11), 3396-3408. https://doi.org/10.1093/brain/awq262

Weissman, D. H., \& Banich, M. T. (2000). The cerebral hemispheres cooperate to perform complex but not simple tasks. Neuropsychology, 14(1), 41-59. https://doi.org/10.1037/0894-4105.14.1.41 
Weng, Y. L., \& Lee, C. L. (2020). Reduced right-hemisphere ERP P600 grammaticality effect is associated with greater right-hemisphere inhibition: Evidence from right-handers with familial sinistrality. Brain Research, 1738, Article 146815. https://doi.org/10.1016/j.brainres.2020.146815

Westerhausen, R., Kreuder, F., Woerner, W., Huster, R. J., Smit, C. M., Schweiger, E., \& Wittling, W. (2006). Interhemispheric transfer time and structural properties of the corpus callosum. Neuroscience Letters, 409(2), 140-145. https://doi.org/10.1016/j.neulet.2006.09.028

Wingfield, A., \& Grossman, M. (2006). Language and the Aging Brain: Patterns of Neural Compensation Revealed by Functional Brain Imaging. Journal of Neurophysiology, 96(6), 2830-2839. https://doi.org/10.1152/jn.00628.2006

Wright, P., Stamatakis, E. A., \& Tyler, L. K. (2012). Differentiating Hemispheric Contributions to Syntax and Semantics in Patients with Left-Hemisphere Lesions. The Journal of Neuroscience, 32(24), 8149. https://doi.org/10.1523/JNEUROSCI.0485-12.2012

Yeh, C., Chen, M.-H., Chen, P.-H., \& Lee, C.-L. (2020). Lateralization as a Symphony: Joint Influence of Interhemispheric Inhibition and Transmission Delay on Brain Asymmetry and Syntactic Processing. PsyArXiv. https://doi.org/10.31234/osf.io/n6q2e 
Zaccarella, E., \& Friederici, A. D. (2017). The neurobiological nature of syntactic hierarchies. Neuroscience \& Biobehavioral Reviews, 81, 205-212.

https://doi.org/10.1016/j.neubiorev.2016.07.038 


\section{Table 1}

Mean grammatical judgment accuracies (\%) for grammatical, ungrammatical, and jointly for both types of phrases (with standard deviations in parentheses) for younger and older adults with right visual field $(\mathrm{RVF} / \mathrm{LH})$ presentation and left visual field (LVF/RH) presentation

\begin{tabular}{cccc}
\hline & & Younger & Older \\
\hline RVF/LH & Grammatical & $90.3(8.1)$ & $79.8(13.0)$ \\
& Ungrammatical & $91.8(8.7)$ & $76.1(15.5)$ \\
& Overall & $91.0(6.7)$ & $77.9(12.4)$ \\
\cline { 2 - 4 } LVF/RH & Grammatical & $86.4(12.8)$ & $79.1(16.0)$ \\
& Ungrammatical & $91.4(10.2)$ & $77.1(15.9)$ \\
& Overall & $88.9(9.0)$ & $78.1(13.9)$ \\
\hline
\end{tabular}




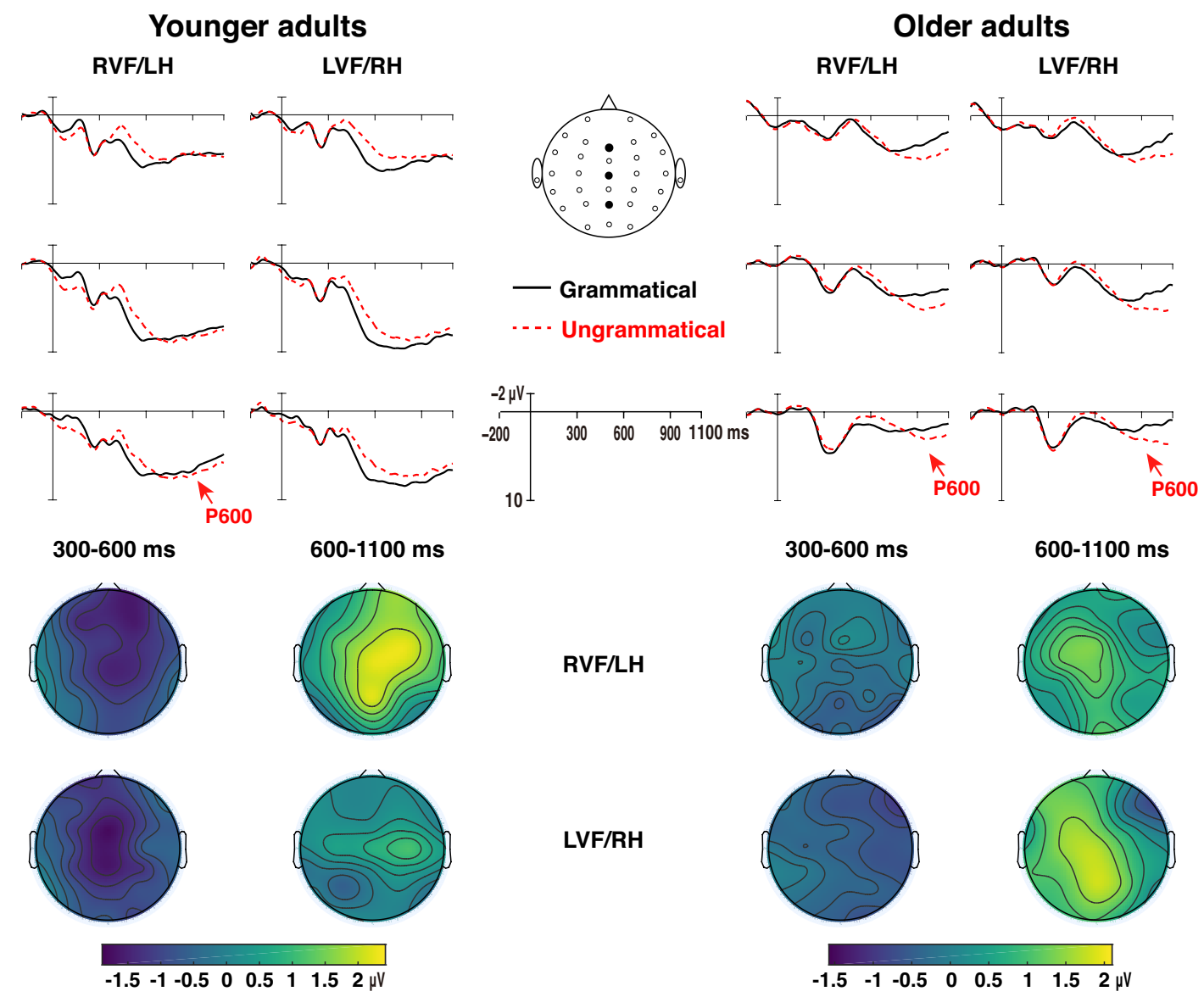

Figure 1. Grand average event-related potentials (ERPs) to the grammatical (the black solid line) and ungrammatical (the red dashed line) critical words with right visual field (RVF/LH) and left visual field $(\mathrm{LVF} / \mathrm{RH})$ presentations plotted for younger (left) and older adults (right) at three representative scalp electrode sites across frontal and posterior regions $(\mathrm{Fz}, \mathrm{Cz}, \mathrm{Pz})$. For this and subsequent figures, positions of the plotted sites are indicated by filled circles on the head diagram (nose at top), negative is plotted up, and data are filtered at $10 \mathrm{~Hz}$ for illustration purposes. The topographic maps show the scalp distributions of mean amplitude differences (Ungrammatical - Grammatical) during the N400 time window (300-600 ms) and peak-to-peak amplitude differences (Ungrammatical - Grammatical) during the P600 time window (600-1100 ms) as a function of visual field for the younger (left) and the older (right) group. 
(A)

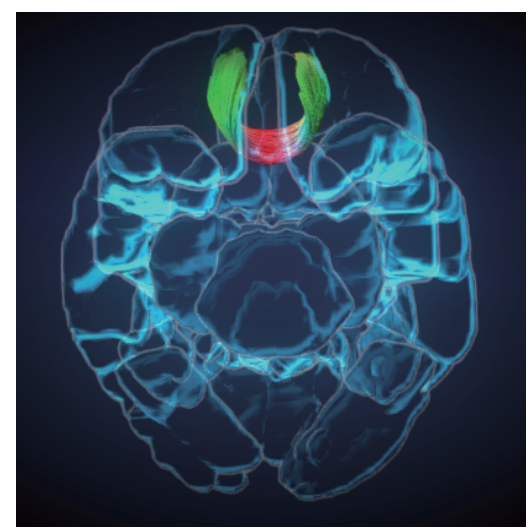

(C)

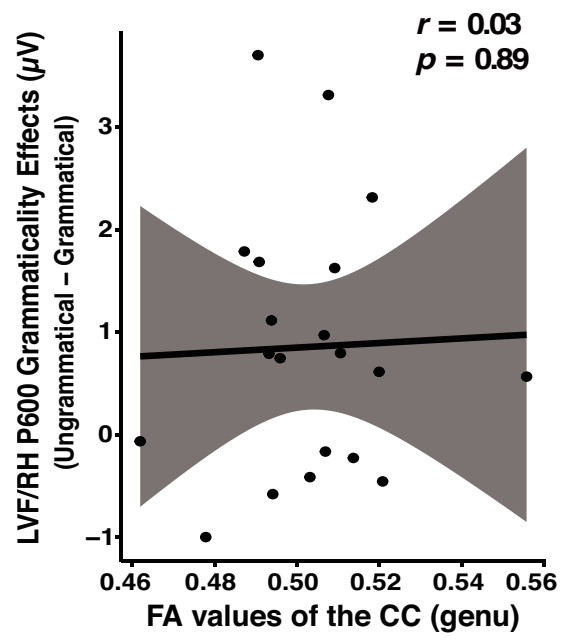

(B)

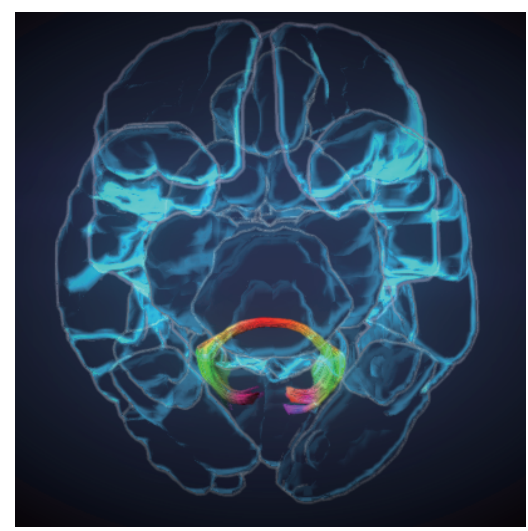

(D)

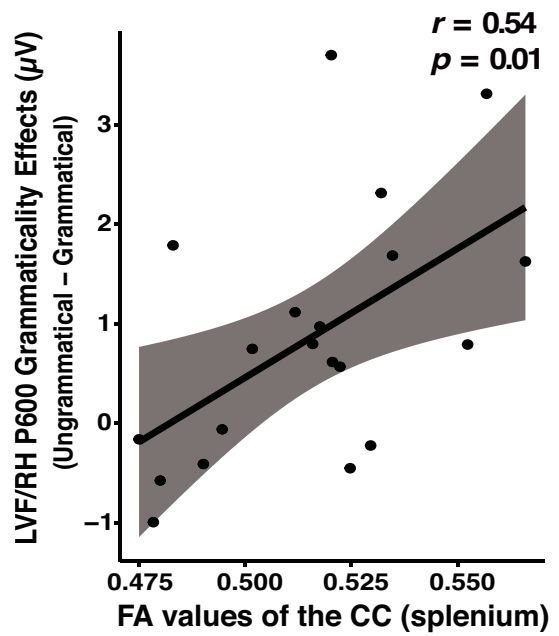

(E)

LVF/RH presentation
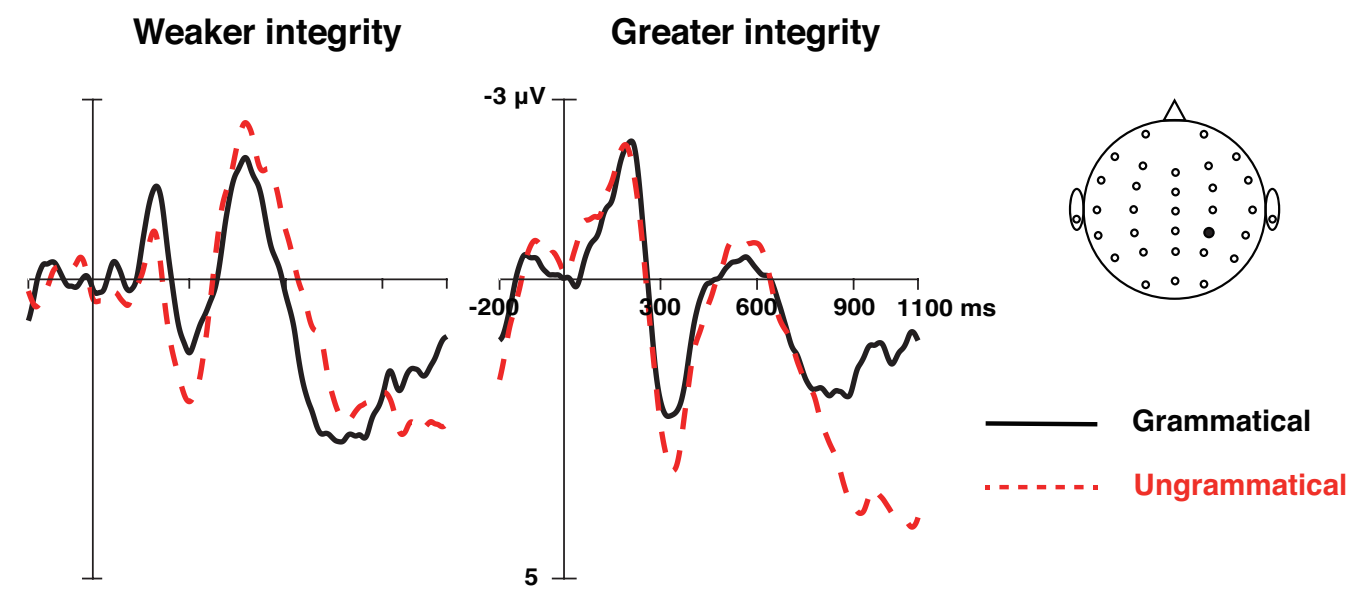

Figure 2. An illustration for (A) the genu and (B) the splenium with an axial view, plotted from Tractatlas (http://abmri.mc.ntu.edu.tw/tractatlas/TBAA_tractatlas.html) based on the NTU-DSI-122 template (Hsu et al., 2015). The front of the head is 
plotted upward. Older adults' microstructural integrity (indexed by FA values) of (C) the genu and (D) the splenium are plotted against the magnitudes of the RH P600 grammaticality effect. (E) Grand average ERPs to the grammatical (the black solid line) and ungrammatical (the red dashed line) critical words with the LVF/RH presentation are overlaid at one representative scalp electrode (CP4) for older adults with weaker (left) and greater (right) splenium integrity based on a median split of FA values for visualization. 


\section{Supplementary materials}

\section{Participants}

All participants were native speakers of Taiwan Mandarin with no exposure to other languages except Taiwan Southern Min before age 5. All were strongly righthanded (mean and range of laterality quotients for younger and older adults: 0.74 $(0.2-1)$ and $0.83(0.6-1))$ and without familial sinistrality background as assessed using the Edinburgh Inventory (Oldfield, 1971) and a familial handedness questionnaire (Lee \& Federmeier, 2015). All had normal or corrected-to-normal vision and no history of psychiatric or neurological disorders or brain damage. Older participants were screened via the Chinese version of Mini-Mental State Examination (MMSE) (Folstein et al., 1975) and all scored within the local normal range, i.e. $>25$ points (Guo et al., 1988).

\section{Materials}

A total of 216 syntactic category unambiguous words (108 nouns and 108 verbs) were used as targets. Their word-class dominancy was determined by the probability of each word being used as the target word class (i.e., a noun or a verb) in the Sinica Corpus (Chen et al., 1996). These words and their corresponding syntactic cues were rated by young native speakers of Taiwan Mandarin (mean age $=23$ years; age range $=19-30$ years) for subjective familiarity ( 1 : very unfamiliar; 7 : very familiar; $\mathrm{N}=39$ ) 
and concreteness ( 1 : very abstract; 7 : very concrete; $N=40)$, and phrases derived from these words were rated for acceptability (1: very ungrammatical; 7: very grammatical; $\mathrm{N}=40$ ) (Supplementary Table 1). Raters were not participants of the main ERP experiment. Additional 192 filler phrases of the same syntactic structure were added, among which 164 were grammatical so ungrammatical trials accounted for one-third of the total trials. Word class dominancy was not systematically controlled for targets in the grammatical fillers. As a result of the prevalence of word-class ambiguous words in Taiwan Mandarin (Huang, Chu-Ren, \& Chang, 2002), a majority of the filler trials $(\mathrm{N}=136)$ could be used as multiple word classes (e.g., 發明 can be used as a noun "invention" or a verb "invent"). The same set of materials was also used in Weng and Lee (2020).

\section{Divided visual field experiment}

We highlight that in addition to the central red dot, the brief $200 \mathrm{~ms}$ presentation of target words minimized participants' eye orientation to the target word before it disappeared. Further, to reduce the likelihood of anticipatory saccades, target words were pseudo-randomly presented to either visual field such that no more than two consecutive trials were presented to the same visual field. Finally, we also monitored participants' horizontal eye movements with the electrooculogram. As past findings showed variations of ERPs for horizontal eye movements due to individual 
differences in the eye movement control and the online positioning of HEO channels, we measured each participant's voltage change for 2-degree horizontal eye movements after the main experiment and excluded any trials with a voltage change exceeding the individualized threshold on the HEO channels (Bourne, 2006).

\section{Validation of VF paradigm}

With the divided VF presentation, laterally-presented stimuli are expected to trigger larger N1 responses and a sustained selection negativity over the hemisphere contralateral to the VF of presentation (Federmeier \& Kutas, 2002; Federmeier et al., 2005; Neville et al., 1982). Supplementary Figure 1 shows this pattern for both age groups. To quantify these effects, for both younger and older adults, mean amplitudes over temporal, posterior and occipital sites (TP7, CP3, CP4, TP8, P7, P3, P4, P8, O1, $\mathrm{O} 2)$ during the $\mathrm{N} 1$ time window $(125-200 \mathrm{~ms})$ and the sustained selection negativity time window (300-900 ms) were tested in an ANOVA with two levels of Visual Field (RVF vs. LVF) and two levels of Electrode sites (left vs. right electrode sites). As expected, both age groups showed significant interactions between the two factors [Young: $F(1,34)=46.26, p<0.001$; Old: $F(1,34)=36.12, p<0.001$ ], with larger N1 responses over electrode sites contralateral to visual field of presentation [RVF stimuli: Young $F(1,34)=35.78, p<0.001 ;$ Old $F(1,34)=26.78, p<0.001 ; \mathrm{LVF}$ stimuli: Young $F(1,34)=27.68, p<0.001 ; \operatorname{Old} F(1,34)=13.11, p<0.001]$. 
Similarly, the results for the sustained selection negativity also showed a significant Visual Field by Electrode sites interaction for both age groups [Young: $F(1,34)=$ 50.45, $p<0.001$; Old: $F(1,34)=133.25, p<0.001]$, with more negative responses over electrode sites contralateral to visual field of presentation [RVF stimuli: Young $F(1,34)=17.93, p<0.001$; Old $F(1,34)=62.83, p<0.001 ;$ LVF stimuli: Young $F(1$, $34)=6.62, p<0.05 ;$ Old $F(1,34)=85.29, p<0.001]$. These results thereby validate the visual presentation for both age groups.

\section{Peak-to-peak measurement for P600 responses}

This approach captures the data pattern of the P600 by correcting for the overlap with the preceding N400 effect that is known to vary across studies (Brouwer et al., 2017) or even participants (Tanner \& Van Hell, 2014) (see Lee et al. (2018) and Steinhauer et al. (1999)). As such, the P600 response was measured as the peak-topeak amplitude difference between peaks of the N400s (local minimums-most negative voltage values preceded and followed by more positive values between 300 $600 \mathrm{~ms}$ ) and peaks of the P600s (local maximums between 600-1100 ms) from all scalp channels. As peak-based measures are sensitive to high-frequency noise, to avoid spurious peaks from high-frequency noise (Luck, 2014), for this analysis the waveforms were additionally filtered with a $5 \mathrm{~Hz}$ low pass filter. 


\section{Supplementary Table 1}

Mean (SD) rating values for phrase grammaticality and lexical features of the syntactic cues and target words for grammatical (G) and ungrammatical (UG) conditions.

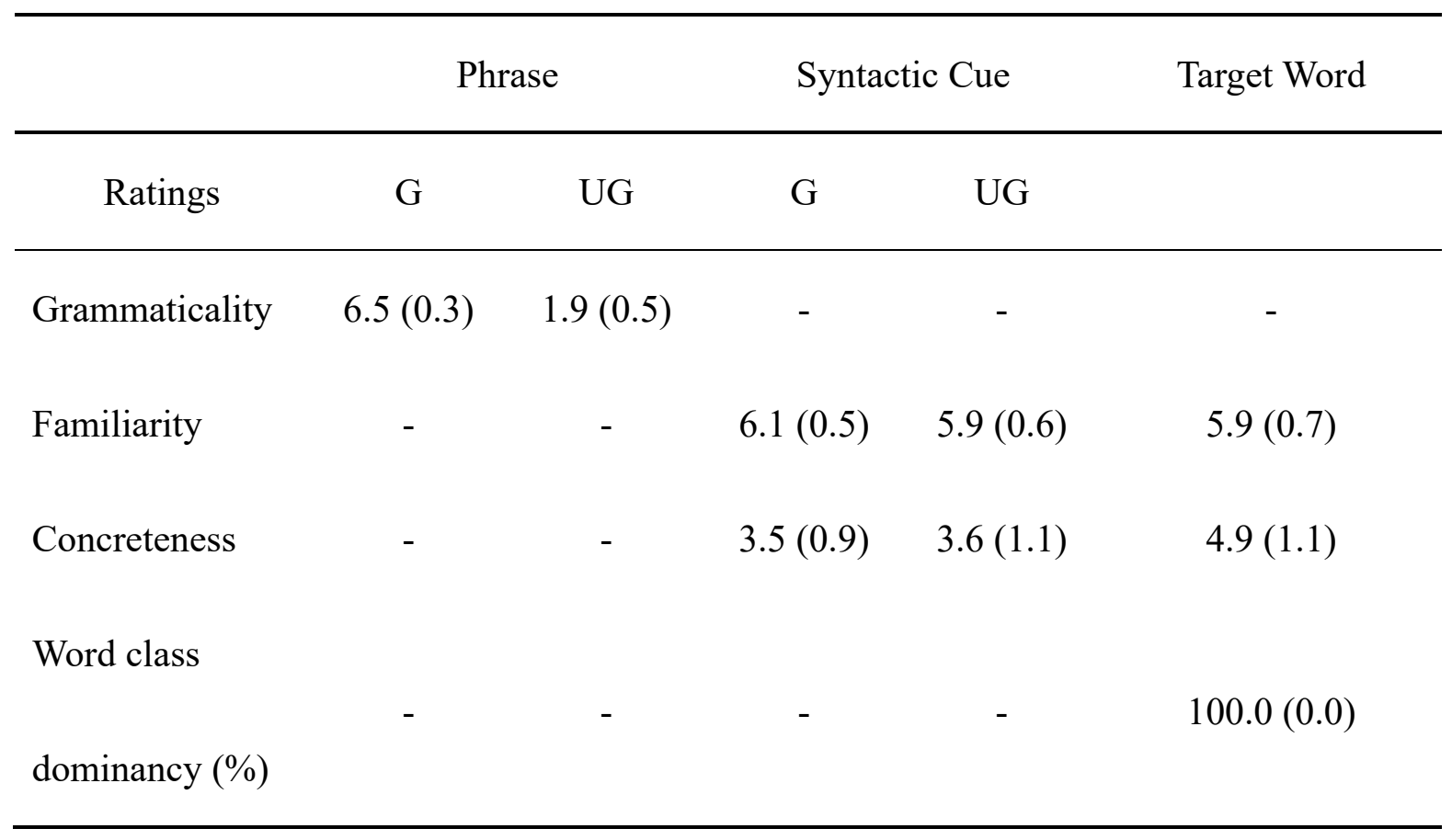


Supplementary Figure 1. Grand average event-related potentials (ERPs) elicited by target words with the left visual field (LVF) presentation (the black solid line) and the right visual field (RVF) presentation (the red dashed line) collapsed across grammaticality at 2 representative posterior sites (P7, P8). The topographic maps show the scalp distributions of mean amplitude differences (LVF - RVF) during the N1 time window (125-200 ms) for the younger (left) and the older (right) group.

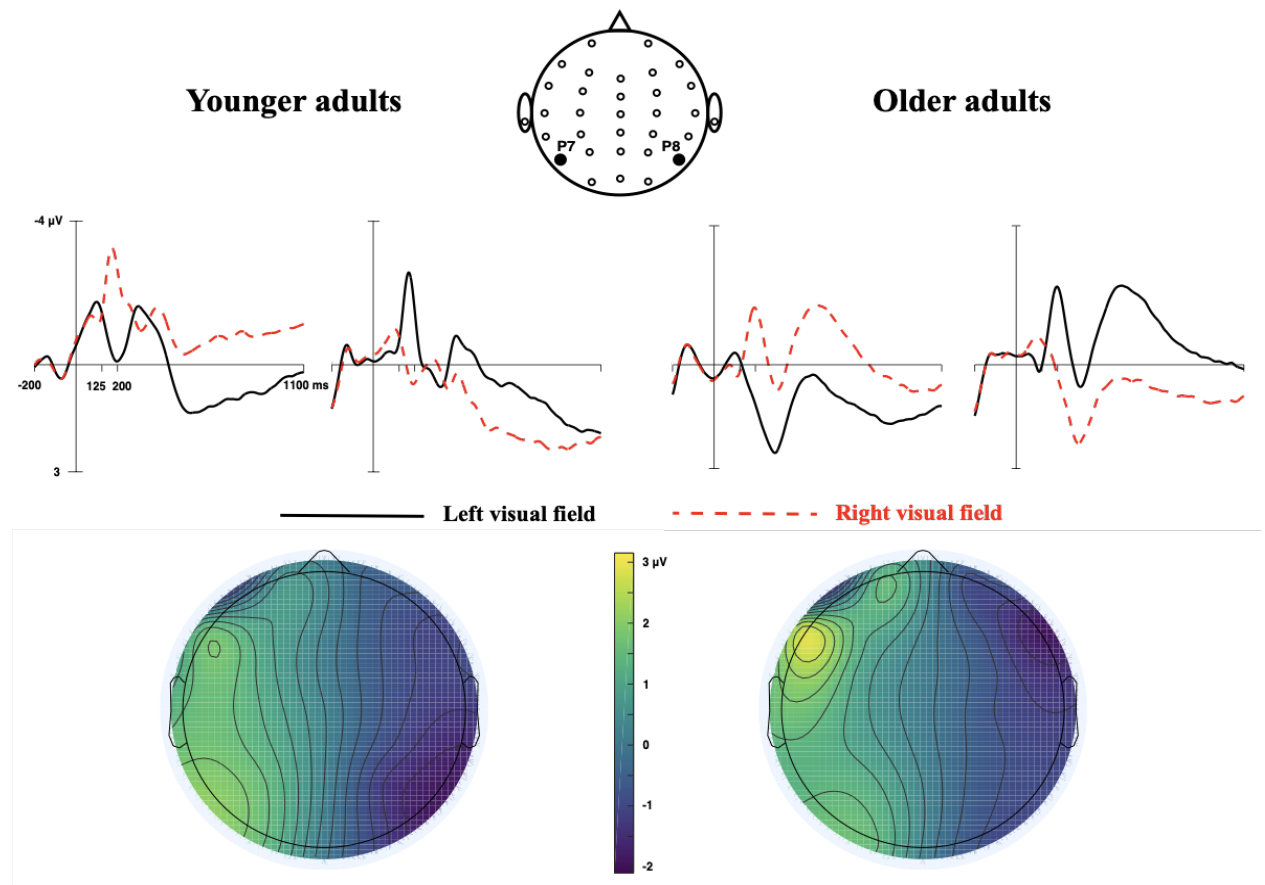


Supplementary Figure 2. In the upper panel, younger adults' RH P600

grammaticality effects are plotted against their microstructural integrity of (A) the genu and (B) the splenium. In the lower panel, younger adults' RH N400

grammaticality effects are plotted against their microstructural integrity of (C) the genu and (D) the splenium.

(A)

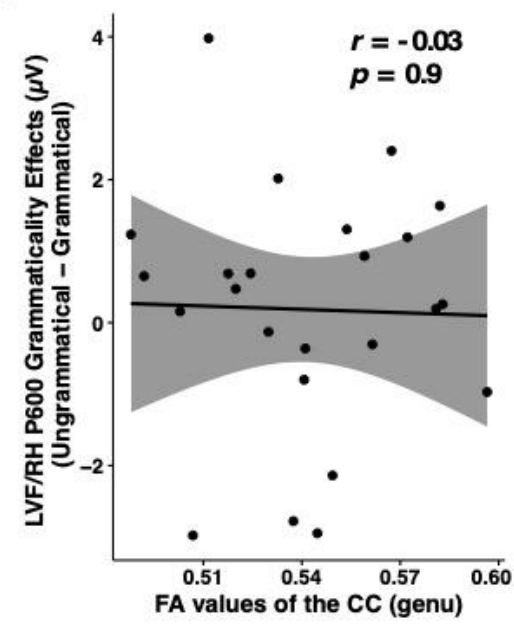

(C)

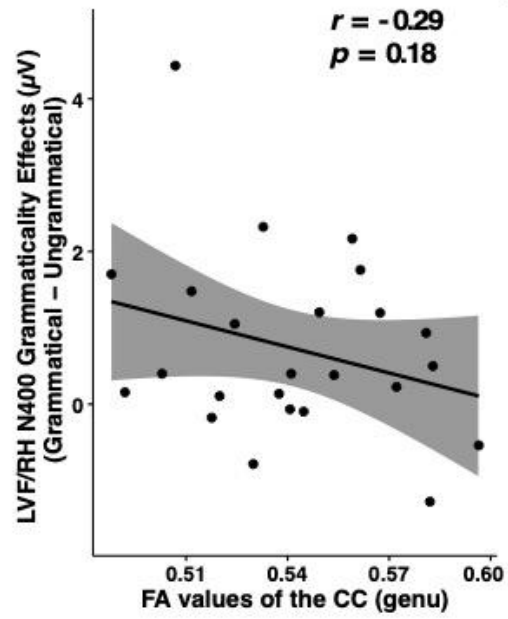

(B)

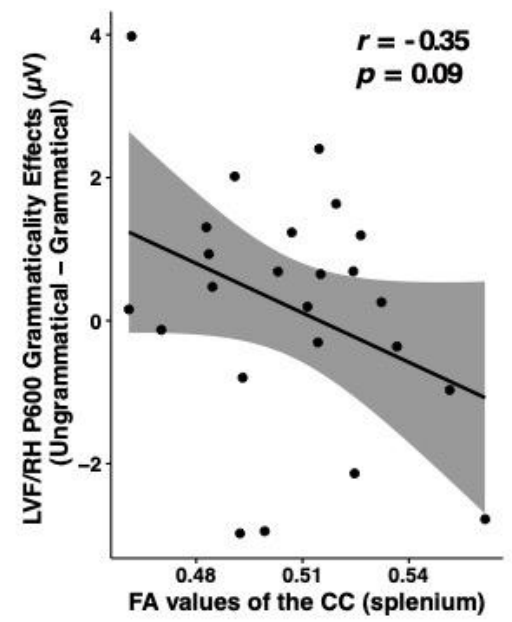

(D)

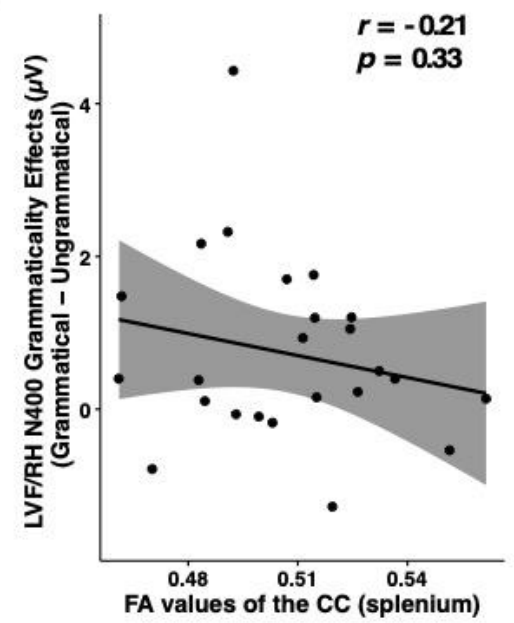


Supplementary Figure 3. Older adults' RH N400 grammaticality effects are plotted against their microstructural integrity of (A) the genu and (B) the splenium.

(A)

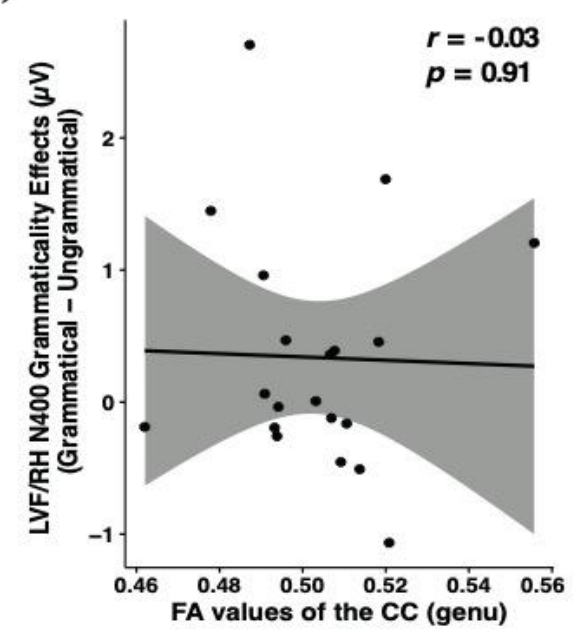

(B)

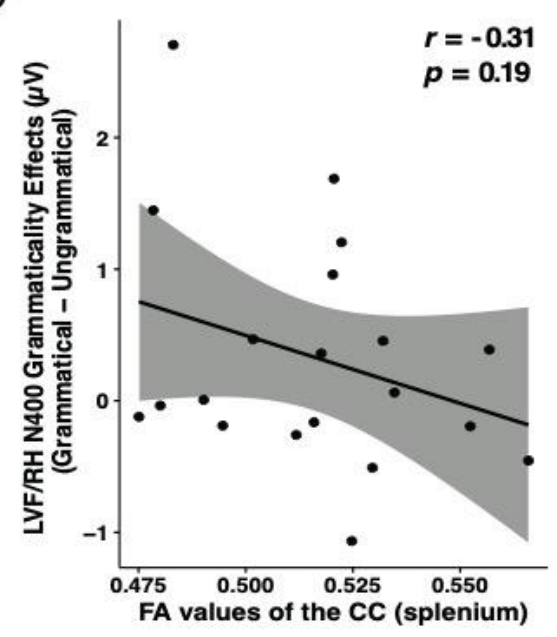




\section{References}

Bourne, V. J. (2006). The divided visual field paradigm: Methodological considerations. Laterality, 11(4), 373-393. https://doi.org/10.1080/13576500600633982

Brouwer, H., Crocker, M. W., Venhuizen, N. J., \& Hoeks, J. C. J. (2017). A Neurocomputational Model of the N400 and the P600 in Language Processing. Cognitive Science, 41(S6), 1318-1352. https://doi.org/10.1111/cogs.12461

Chen, K.-j., Huang, C.-R., Chang, L.-p., \& Hsu, H.-L. (1996). Sinica Corpus: Design Methodology for Balanced Corpora. In B.-S. Park \& J. B. Kim (Eds.), Proceeding of the 11 th Pacific Asia Conference on Language, Information and Computation (pp. 167-176). https://aclanthology.org/Y96-1018

Federmeier, K. D., \& Kutas, M. (2002). Picture the difference: electrophysiological investigations of picture processing in the two cerebral hemispheres. Neuropsychologia, 40(7), 730-747. https://doi.org/10.1016/S00283932(01)00193-2

Federmeier, K. D., Mai, H., \& Kutas, M. (2005). Both sides get the point: Hemispheric sensitivities to sentential constraint. Memory \& Cognition, 33(5), 871-886. https://doi.org/10.3758/BF03193082

Folstein, M. F., Folstein, S. E., \& McHugh, P. R. (1975). "Mini-mental state": a practical method for grading the cognitive state of patients for the clinician. Journal of psychiatric research, 12(3), 189-198. https://doi.org/10.1016/0022$\underline{3956(75) 90026-6}$

Guo, N. W., Liu, H. C., Wong, P. F., Liao, K. K., Yan, S. H., Lin, K. P., Chang, C. Y., \& Hsu, T. C. (1988). Chinese Version and Norms of the Mini-Mental State Examination. Journal Chinese Rehabilitative Medicine(16), 52-59. https://www.airitilibrary.com/Publication/alDetailedMesh?docid=10253009$\underline{\text { 198812-x-16-52-59-a\#Altmetrics }}$

Lee, C. L., \& Federmeier, K. D. (2015). It's All in the Family: Brain Asymmetry and Syntactic Processing of Word Class. Psychological Science, 26(7), 997-1005. 
https://doi.org/10.1177/0956797615575743

Lee, C. L., Huang, H.-W., Federmeier, K. D., \& Buxbaum, L. J. (2018). Sensory and semantic activations evoked by action attributes of manipulable objects: Evidence from ERPs. Neuroimage, 167, 331-341. https://doi.org/10.1016/j.neuroimage.2017.11.045

Luck, S. J. (2014). An introduction to the event-related potential technique. MIT press.

Neville, H. J., Kutas, M., \& Schmidt, A. (1982). Event-related potential studies of cerebral specialization during reading: I. Studies of normal adults. Brain and language, 16(2), 300-315. https://doi.org/10.1016/0093-934X(82)90088-8

Oldfield, R. C. (1971). The assessment and analysis of handedness: the Edinburgh inventory. Neuropsychologia, 9(1), 97-113. https://doi.org/10.1016/00283932(71)90067-4

Steinhauer, K., Alter, K., \& Friederici, A. D. (1999). Brain potentials indicate immediate use of prosodic cues in natural speech processing. Nature Neuroscience, 2(2), 191-196. https://doi.org/10.1038/5757

Tanner, D., \& Van Hell, J. G. (2014). ERPs reveal individual differences in morphosyntactic processing. Neuropsychologia, 56, 289-301. https://doi.org/10.1016/j.neuropsychologia.2014.02.002

Weng, Y. L., \& Lee, C. L. (2020). Reduced right-hemisphere ERP P600 grammaticality effect is associated with greater right-hemisphere inhibition: Evidence from right-handers with familial sinistrality. Brain Research, 1738, Article 146815. https://doi.org/10.1016/j.brainres.2020.146815 